\title{
Motor-driven restructuring of cytoskeleton composites leads to tunable time-varying elasticity
}

Janet Y. Sheung ${ }^{1 *}$, Daisy H. Achiriloaie ${ }^{1}$, Christopher Currie ${ }^{2}$, Karthik Peddireddy ${ }^{2}$, Aaron Xie ${ }^{1}$, Jessalyn Simon-Parker ${ }^{1}$, Gloria Lee ${ }^{2}$, Michael J. Rust ${ }^{3}$, Moumita Das ${ }^{4}$, Jennifer L. Ross ${ }^{5}$, Rae M. Robertson-Anderson ${ }^{2 *}$

${ }^{1}$ W. M. Keck Science Department, Scripps College, Pitzer College, and Claremont McKenna College, 925 N. Mills Ave. Claremont CA 91711, USA

${ }^{2}$ Department of Physics and Biophysics, University of San Diego, 5998 Alcala Park, San Diego, CA, 92110, USA

${ }^{3}$ Department of Molecular Genetics and Cell Biology, University of Chicago, Chicago, IL 60637, USA.

${ }^{4}$ School of Physics and Astronomy, Rochester Institute of Technology, Rochester, NY 14623, USA.

${ }^{5}$ Department of Physics, Syracuse University, Syracuse, NY 13244, USA.

*jsheung@kecksci.claremont.edu, randerson@sandiego.edu

\section{Supporting Information}

\section{Section S1. Materials and Methods}

Composite Preparation: Rabbit skeletal actin monomers, porcine brain tubulin dimers, rhodamine-labeled tubulin dimers, and rabbit skeletal myosin dimers (Cytoskeleton AKL99, T240, TL590M, MY02) are flash frozen in experimental-sized aliquots and stored at $-80^{\circ} \mathrm{C} .{ }^{1}$ Myosin is stored with $1.25 \mathrm{M} \mathrm{KCl}$ to prevent minifilament polymerization. Immediately prior to use, enzymatically dead myosin dimers are removed from myosin II aliquots using an actin spin-down protocol previously described. ${ }^{1}$ Active actin-microtubule composites are prepared as described previously. ${ }^{2}$ In brief, actin and microtubules are polymerized together from a total of $5.8 \mu \mathrm{M}$ of actin monomers and tubulin dimers in PEM-100 (100 mM PIPES, 2 mM MgCl 2,2 mM EGTA) supplemented with $0.1 \%$ Tween, $4 \mathrm{mM}$ ATP, $4 \mathrm{mM}$ GTP, phalloidin concentration matching the total actin concentration, and $5 \mu \mathrm{M}$ Taxol. ${ }^{3}$ The specific molar concentrations of actin and tubulin depend on the actin-tubulin molar percentages desired (25-75, 50-50, or 75-25). To image microtubules, $15 \%$ of tubulin monomers are labeled with rhodamine. After the composites polymerize for 30 minutes at $37^{\circ} \mathrm{C}$, an oxygen scavenging system $(45 \mu \mathrm{g} / \mathrm{ml}$ glucose, $0.005 \% \beta$ mercaptoethanol, $43 \mu \mathrm{g} / \mathrm{ml}$ glucose oxidase, $7 \mu \mathrm{g} / \mathrm{ml}$ catalase) is added to reduce photobleaching during fluorescence imaging, $0.24 \mu \mathrm{M}$ myosin dimers are added to introduce network activity, and $50 \mu \mathrm{M}$ blebbistatin is added to inhibit myosin-actin interactions until activation via $488 \mathrm{~nm}$ illumination using the optical tweezers (Fig 1A). Myosin dimers polymerize into minifilaments upon addition to the composite due to the drop in $\mathrm{KCl}$ concentration.

Microrheology Measurements: We embed a sparse amount of 4.5- $\mu \mathrm{m}$ diameter microspheres (beads; Polysciences) to composites to facilitate linear and nonlinear microrheology measurements (Fig 1C,D). After polymerization, we pipette the protein and bead mixture into a sample chamber made from a glass slide and silanized coverslip, separated by $\sim 100 \mu \mathrm{m}$ with double-sided tape to accommodate $\sim 10 \mu \mathrm{L}$, and immediately sealed with epoxy to prevent evaporation. Beads are coated with BSA (ThermoFisher), following previously described 
methods, ${ }^{4}$ to inhibit binding interactions with the proteins. The protocol for silanizing the coverslips is described elsewhere. ${ }^{1}$

The force-measuring optical tweezers is custom-built around an Olympus IX71 fluorescence microscope frame to enable epifluorescence imaging, as previously thoroughly described. ${ }^{5,6}$ In brief, a $1064 \mathrm{~nm} \mathrm{Nd:YAG} \mathrm{fiber} \mathrm{laser} \mathrm{(Manlight)} \mathrm{is} \mathrm{focused} \mathrm{with} \mathrm{a} \mathrm{60×1.4} \mathrm{NA} \mathrm{objective} \mathrm{(Olympus).}$ A position-sensing detector (Pacific Silicon Sensor) measures the deflection of the trapping laser, which is proportional to the force acting on the trapped microsphere. We use Stokes drag method to calibrate trap stiffness. ${ }^{7}$ A nanopositioning piezoelectric stage (Mad City Labs) moves the trapped microsphere relative to the sample (Fig 1C,D). We record the stage position and laser deflection at $20 \mathrm{kHz}$ using custom-written Labview codes.

For linear microrheology measurements (Figs 1C, 2), we record the force of an optically trapped microsphere in $\mathrm{x}$ - and y-directions, $F_{x}(t)$ and $F_{y}(t)$, for $360 \mathrm{~s}$. We repeat measurements for beads ( 6 before activity, 17 during activity) located at different regions of the sample chamber separated by $>300 \mu \mathrm{m}$. Previously, we showed that actin filaments and microtubules in the fastest myosindriven composite studied here (75-25) contract at an average velocity of $v \approx 35 \pm 3 \mathrm{~nm} / \mathrm{s}$. ${ }^{1}$ From this speed we compute an effective strain rate $\dot{\gamma}_{\text {active }}$ and frequency $\omega_{\text {active }}=2 \pi \dot{\gamma}_{\text {active }}$ for the strain exerted on the trapped bead of radius $R$ by the motor-driven composite during motor-driven contraction via $\dot{\gamma}=3 v / \sqrt{2} R .^{3}$ Within this framework, $\omega_{\text {active }} \approx 0.18 \mathrm{rad} / \mathrm{s}$, so we can approximate that our system is in quasi-steady-state for $\omega \approx 1 \mathrm{rad} / \mathrm{s}\left(\sim 6 \mathrm{x}\right.$ higher than $\left.\omega_{\text {active }}\right)$. This approximation allows the use of the Generalized Stokes-Einstein Relation (GSER) to derive the elastic and viscous moduli, $G^{\prime}(\omega)$ and $G^{\prime \prime}(\omega)$, from the force data for $\omega>1 \mathrm{rad} / \mathrm{s}$, as follows.

From $F_{x}(t)$, we compute the normalized mean-square displacements $\pi(\tau)=\left[<r^{2}(\tau)\right\rangle / 2<$ $r^{2}>$ ] then transform these into Fourier space, $-\omega^{2} \pi(\omega)\left(1-e^{-i \omega \tau_{1}}\right) \frac{\pi\left(\tau_{1}\right)}{\tau_{1}}+\pi_{\infty} e^{-i \omega \tau_{N}}+$ $\sum_{k=2}^{N}\left(\frac{\pi_{K}-\pi_{K-1}}{\tau_{K}-\tau_{K-1}}\right)\left(e^{-i \omega t_{K-1}}-e^{-i \omega t_{K}}\right)$. Here $\tau$ is the lag time, 1 and $N$ represent the first and last points of $\pi(\omega)$, which is oversampled via interpolation using the MATLAB function pchip. GSER then gives the complex modulus $G^{*}=G^{\prime}(\omega)+i G^{\prime \prime}(\omega)=\left(\frac{k}{6 \pi R}\right)\left(\frac{1}{i \omega \pi(\omega)}-1\right)$ where $R$ is the microsphere radius and $k$ is the calibrated trap stiffness.

For nonlinear microrheology measurements (Figs 1D, 3), we use a piezoelectric nanopositioning stage to displace a trapped microsphere $5 \mu \mathrm{m}$ at a constant speed of $5 \mu \mathrm{m} / \mathrm{s}$ relative to the sample chamber. For each trial, we record the force exerted on the bead for 5 seconds prior to the strain (equilibrium), during the $1 \mathrm{~s}$ strain (strain), and $15 \mathrm{~s}$ after the strain (relaxation). We repeat measurements for beads ( 21 before activity, 18 after activity) located at different regions of the sample chamber each separated by $500 \pm 100 \mu \mathrm{m}$. Custom-written MATLAB scripts are used for post-acquisition data analysis, and OriginPro is used to fit force relaxation curves to two-term exponentials $F(t)=F_{\infty}+C_{1} e^{-t / \tau_{1}}+C_{2} e^{-t / \tau_{2}}$. Error bars shown in Figure 3 are determined by computing the standard error in values for 5-7 random subsets of the data.

Confocal Microscopy: To image the structure of the composites over time, we acquired fluorescence confocal images of rhodamine-labeled microtubules in the composite using a Nikon A1R laser scanning confocal microscope with a $60 \times 1.4$ NA objective (Nikon), $561 \mathrm{~nm}$ laser, and 
$561 / 595 \mathrm{~nm}$ excitation/emission filters. The 561 laser is set at $5 \%$ intensity for a pixel dwell of 2.2. We have previously shown that illumination with this laser line and filter set shows no signs of blebbistatin deactivation (i.e. motor activation). ${ }^{1} 512 \times 512$ pixel images $(213 \mu \mathrm{m} \times 213 \mu \mathrm{m})$ are collected at different points in the sample in the middle of the $\sim 70 \mu \mathrm{m}$ thick chamber at each time interval (before activation of myosin II motors, 2 hours after, 4 hours after, and 8 hours after) (Fig 4, Fig S1). Activation is achieved by illuminating the sample at $488 \mathrm{~nm}$ for $10 \mathrm{mins}$, to deactivate blebbistatin, using the Olympus IX71 on the optical tweezers.

Spatial Image Autocorrelation (SIA): We use custom Python scripts ${ }^{8}$ to perform spatial image autocorrelation (SIA) analysis on each of five confocal images of rhodamine-labeled microtubules in the actin-microtubule composites acquired at different time points relative to myosin activation: before, $+2 \mathrm{hrs},+4 \mathrm{hrs}$ and $+8 \mathrm{hrs}$. SIA measures the correlation in intensity $g(r)$ of two pixels in an image as a function of separation distance $r$. We generate autocorrelation curves by taking the fast Fourier transform of an image at the given time, multiplying by its complex conjugate, and then applying an inverse Fourier transform and normalizing by the squared intensity:

$$
g(r)=\frac{F^{-1}\left(|F(I(r))|^{2}\right)}{[I(r)]^{2}}
$$

The correlation length $\xi$ is determined by fitting the decaying section of each autocorrelation curve to an exponential: $g(r) \propto e^{\frac{-r}{\xi}}$.

\section{References}

(1) Lee, G.; Leech, G.; Rust, M. J.; Das, M.; McGorty, R. J.; Ross, J. L.; Robertson-Anderson, R. M. Myosin-Driven Actin-Microtubule Networks Exhibit Self-Organized Contractile Dynamics. Sci. Adv. 2021, 7 (6), eabe4334. https://doi.org/10.1126/sciadv.abe4334.

(2) Ricketts, S. N.; Ross, J. L.; Robertson-Anderson, R. M. Co-Entangled Actin-Microtubule Composites Exhibit Tunable Stiffness and Power-Law Stress Relaxation. Biophys. J. 2018, 115 (6), 1055-1067. https://doi.org/10.1016/j.bpj.2018.08.010.

(3) Ricketts, S. N.; Francis, M. L.; Farhadi, L.; Rust, M. J.; Das, M.; Ross, J. L.; Robertson-Anderson, R. M. Varying Crosslinking Motifs Drive the Mesoscale Mechanics of Actin-Microtubule Composites. Sci. Rep. 2019, 9 (1), 12831. https://doi.org/10.1038/s41598-019-49236-4.

(4) Gurmessa, B.; Francis, M.; Rust, M. J.; Das, M.; Ross, J. L.; Robertson-Anderson, R. M. Counterion Crossbridges Enable Robust Multiscale Elasticity in Actin Networks. Phys. Rev. Res. 2019, 1 (1), 013016. https://doi.org/10.1103/PhysRevResearch.1.013016.

(5) Robertson-Anderson, R. M. Optical Tweezers Microrheology: From the Basics to Advanced Techniques and Applications. ACS Macro Lett. 2018, 7 (8), 968-975. https://doi.org/10.1021/acsmacrolett.8b00498.

(6) Peddireddy, K. R.; Lee, M.; Schroeder, C. M.; Robertson-Anderson, R. M. Viscoelastic Properties of Ring-Linear DNA Blends Exhibit Nonmonotonic Dependence on Blend Composition. Phys. Rev. Res. 2020, 2 (2), 023213. https://doi.org/10.1103/PhysRevResearch.2.023213.

(7) Brau, R. R.; Ferrer, J. M.; Lee, H.; Castro, C. E.; Tam, B. K.; Tarsa, P. B.; Matsudaira, P.; Boyce, M. C.; Kamm, R. D.; Lang, M. J. Passive and Active Microrheology with Optical Tweezers. J. Opt. Pure Appl. Opt. 2007, 9 (8), S103-S112. https://doi.org/10.1088/1464-4258/9/8/S01.

(8) McGorty, R. Rmcgorty/Image-Correlation; 2020. 

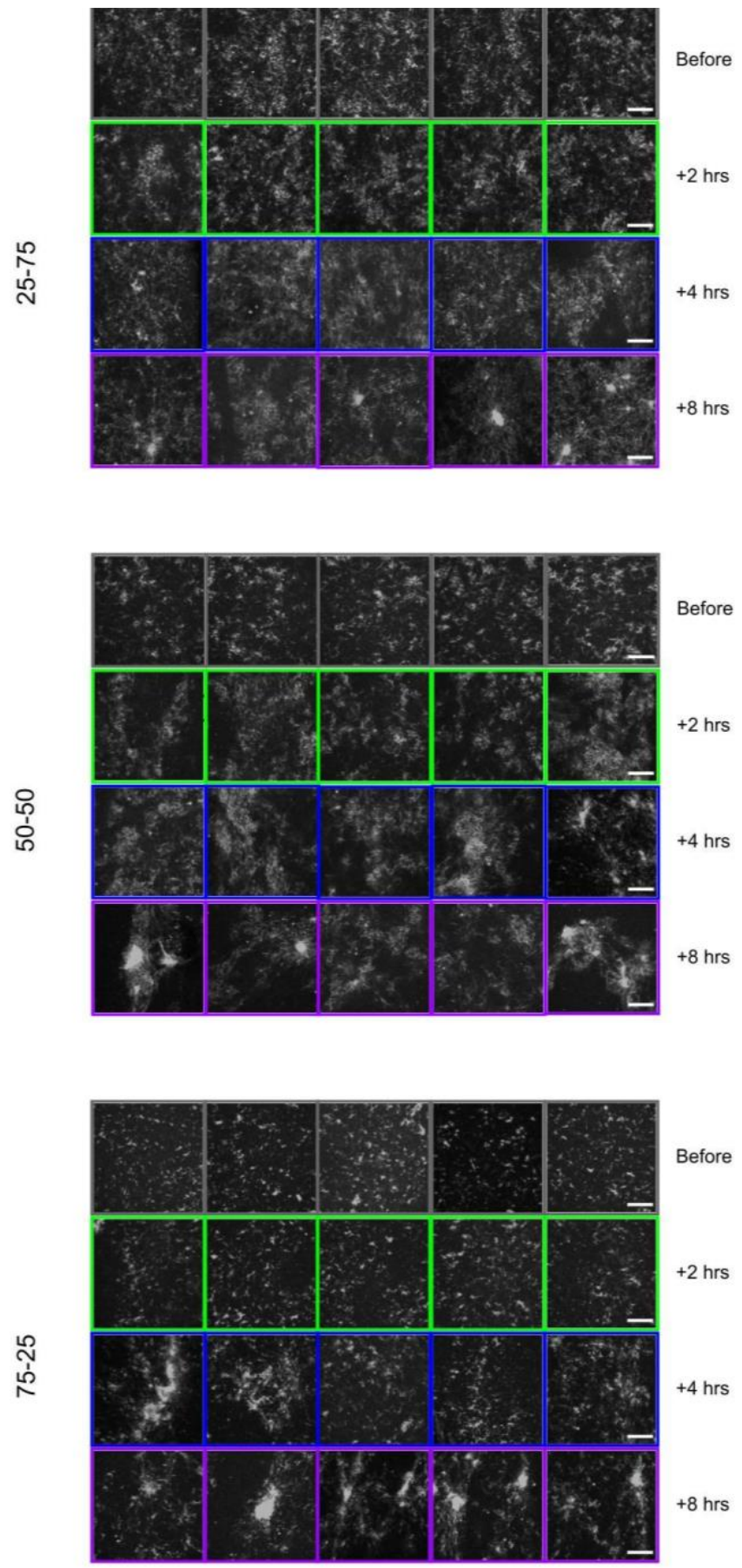

Figure S1. Fluorescence confocal images of microtubules in myosin-driven actinmicrotubule composites show clustering and increased heterogeneity sustained hours after activation. Images of rhodamine-labeled microtubules at 5 different locations in 25-75 (top), 50-50 (middle), and 75-25 (bottom) actin-microtubule composites at varying time points relative to myosin activation (listed to the right of each row). Each set of five images is used in the spatial image autocorrelation analysis shown in Figure 4. Color coding matches that in Figs 2-4. $512 \times 512$ pixel grayscale images are collected as described in Methods and each row shares the scale bar on the right, indicating $50 \mu \mathrm{m}$. 


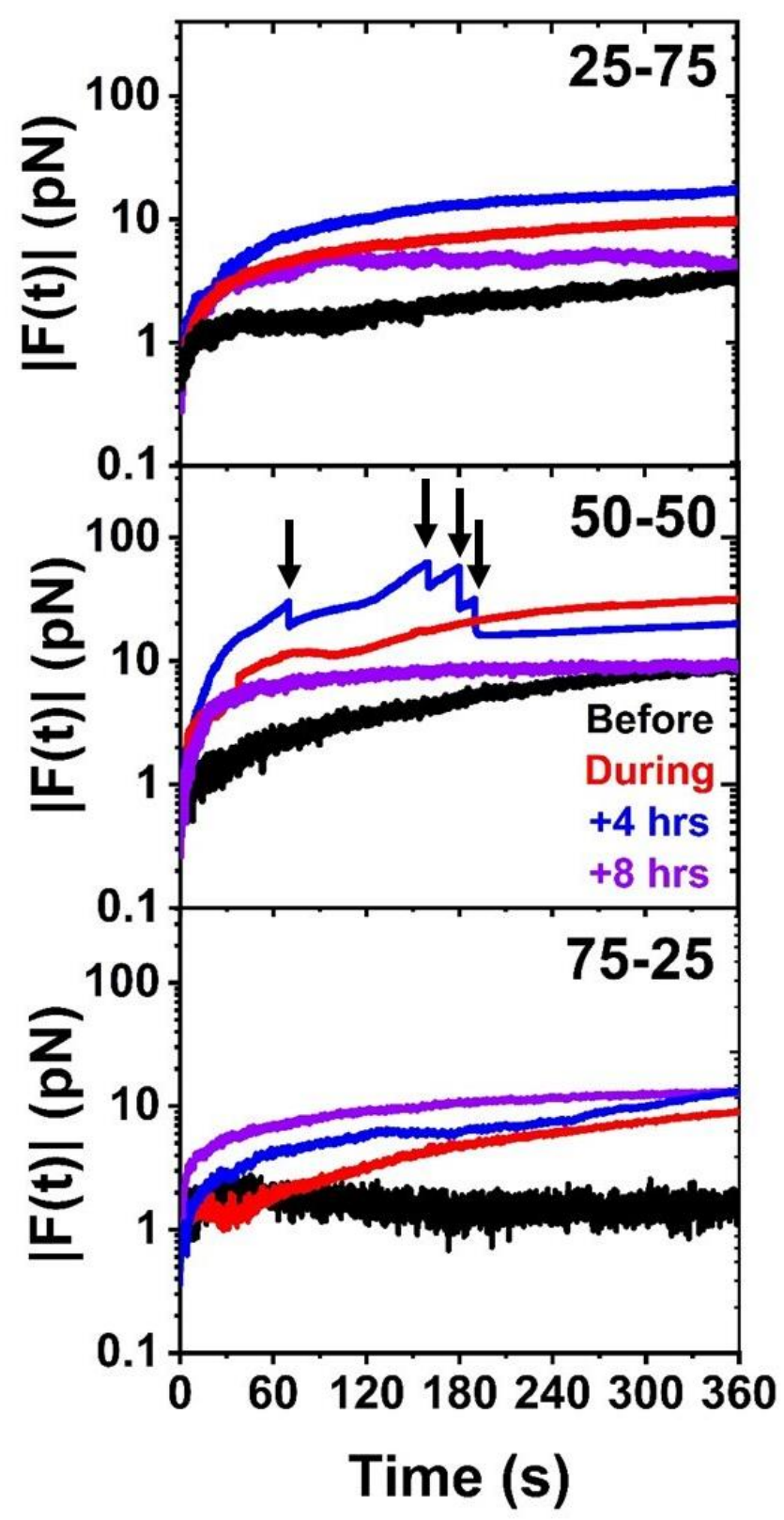

Figure S2. Force exerted on optically trapped microspheres, $|F(t)|=\left(F_{x}^{2}+F_{y}^{2}\right)^{1 / 2}$, embedded in active actin-microtubule composites. The different panels correspond to different actin-tubulin molar percentages (listed in each panel), and the different curves correspond to the average force measured before (black) and during (red) 3 minutes of myosin activation, as well as 4 hours (blue) and 8 hours (purple) after. At 4 hours, the force exerted by the 50-50 composite exceeded the strength of the optical trap in some cases, so the bead was lost before the end of the measurement. Arrows above this force curve indicate locations where the number of individual trials contributing to the average decreases due to truncated trials. Data is the same as shown in Fig 2A but plotted on a log-y scale. 